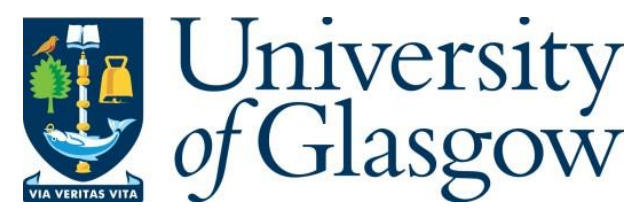

Hou, C., Metcalfe, N. B. and Salin, K. (2021) Is mitochondrial reactive oxygen species production proportional to oxygen consumption? A theoretical consideration. BioEssays, 43(4), 2000165. (doi: 10.1002/bies.202000165).

There may be differences between this version and the published version. You are advised to consult the publisher's version if you wish to cite from it.

This is the peer reviewed version of the following article:

Hou, C., Metcalfe, N. B. and Salin, K. (2021) Is mitochondrial reactive oxygen species production proportional to oxygen consumption? A theoretical consideration. BioEssays, 43(4), 2000165, which has been published in final form at 10.1002/bies.202000165. This article may be used for non-commercial purposes in accordance with Wiley Terms and Conditions for Self-Archiving.

http://eprints.gla.ac.uk/234339/

Deposited on: 16 April 2021

Enlighten - Research publications by members of the University of Glasgow http://eprints.gla.ac.uk 
$6{ }^{1}$ Department of Biological Sciences, Missouri University of Science and Technology, Rolla,

$7 \quad$ U.S.A. 65409

$8{ }^{2}$ Institute of Biodiversity, Animal Health and Comparative Medicine, Graham Kerr Building,

9 University of Glasgow, Glasgow G12 8QQ United Kingdom

$10{ }^{3}$ Ifremer, Unité de Physiologie Fonctionnelle des Organismes Marins - Marine Environmental

11 Science Laboratory, LEMAR UMR 6539, BP70, Plouzané, 29280, France

12

$13 *$ Corresponding author's email: houch@mst.edu

\section{Is Mitochondrial Reactive Oxygen Species Production Proportional to Oxygen consumption? A Theoretical Consideration}

\author{
Chen Hou ${ }^{1 *}$, Neil B. Metcalfe ${ }^{2}$, Karine Salin ${ }^{3}$
} that mitochondria operate in the different respiration states. The model suggests that under three

14

\section{Abstract}

It has been assumed that at the whole organismal level, the mitochondrial Reactive Oxygen Species (ROS) production is proportional to the oxygen consumption. Recently, a number of researchers have challenged this assumption, based on the observation that the ROS production per unit oxygen consumed in the resting state of mitochondrial respiration is much higher than that in the active state. Here, we develop a simple model to investigate the validity of the assumption and the challenge of it. The model highlights the significance of the time budget physiologically possible conditions, the difference in ROS production per unit oxygen consumed 
24 between the respiration states does not upset the proportionality between the whole animal ROS

25 production and oxygen consumption. The model also shows that mitochondrial uncoupling

26 generally enhances the proportionality.

27

28 Key words: mitochondria, variation, respiration states, uncoupling, theoretical model

29 


\section{Introduction}

Mitochondria are the major site for both ATP and reactive oxygen species (ROS)

32 production. When produced in excess for antioxidants to keep the steady state concentration in balance, ROS cause oxidative damage to cellular lipids, proteins and DNA, and so become a major contributing factor for oxidative damage and aging [1-5], although some studies have shown extension of lifespan by mildly increasing ROS concentration, perhaps through hermetic mechanisms [6].

Mitochondrial ROS production rate can vary significantly both between and within individuals [7-9]. The "rate of living theory of aging" and its modern version, "the oxidative stress theory of aging", assume a proportional relationship between ROS production and oxygen consumption at the whole organismal level [5,10-12]. This assumption has been challenged by a number of researchers (e.g., $[1,13,14])$. The challengers noticed the significant difference in the

42 rates of ROS production per unit oxygen consumed between the resting and the active states of 43 mitochondrial respiration, i.e. the minimal and maximal rates of Complex IV activity. ROS

44 production depends on the redox state of the electron transfer chain (ETC) and proton motive 45 force (PMF) across the inner mitochondrial membrane created by the pumping out of protons by the mitochondrial respiratory chain complexes $[13,15]$. PMF is positively correlated with

47 membrane potential and the gradient of proton concentration [16]. In the resting respiration rate 48 (State 4, the non-phosphorylating state) of the mitochondria, the PMF and membrane potential 49 are high compared to the active state (State 3, the phosphorylating state). This condition causes a 50 high rate of ROS production [17]. In contrast, during periods of active respiration when ATP is 51 being synthesized at a high rate, the elevated oxygen consumption and decreased oxygen partial 52 pressure cause a reduction in the rate of ROS production (as described in Fig. 1 in [17]). 
Another factor that affects the difference in ROS production between the resting and

54 active states is mitochondrial uncoupling. During oxidative phosphorylation, the leakage of

55 protons across the mitochondrial inner membrane leads to uncoupling, in which protons bypass

56 the ATP synthase molecule and so shortcut the coupling of substrate oxidation to the

57 phosphorylation of ADP to produce ATP [18]. By reducing PMF, the uncoupling process

58 decreases the rate of ROS production [18]. However, the uncoupling-induced reductions of ROS

59 production are different in the resting and the active states. In the resting state, where PMF is

60 high, the production of ROS is extremely sensitive to the strength of the membrane potential, i.e.,

61 a slight uncoupling, which causes a slight reduction in potential, causes a substantial reduction in

62 ROS production. In contrast, in the active state, where PMF is low, the ROS production is not as

63 sensitive to the membrane potential as in the resting state, so in the active state the same degree

64 of uncoupling causes relatively little reduction in the ROS production [19].

65 Due to the concerted effects of these factors, the ROS production per unit oxygen

66 consumed (denoted as ROS/Oxy hereafter) is substantially different between the resting and the

67 active. It is noteworthy that for one unit of oxygen consumed, ROS production in the resting

68 state can be as much as 10 times higher than in the active state $[13,19]$, i.e., from the resting state

69 to the active states there can be no or even negative correlation between the ROS production and

70 oxygen consumption. Because of these observations, some researchers claimed that "live fast

71 and die young," the notion underlying the "rate of living theory of aging" [20] is wrong, and

72 should be abandoned [1,13]. (Note: This statement mainly applies for mitochondrial

73 contributions, because mitochondria are not the only source of ROS. They consume $90 \%$ of the

74 oxygen uptake by animals. In this study, we do not consider the other $10 \%$ non-mitochondrial

75 oxygen consumption, because it is not linked to variation in mitochondrial function.) 
In this essay, we test the validity of the claim that whole organismal ROS production rate

77 is proportional to oxygen consumption rate by a simple theoretical model, which is tautological,

78 but allows evaluation of how ROS production varies with oxygen consumption under different

79 levels of mitochondrial activity. We also discuss how mitochondrial uncoupling affects the

80 proportionality at the whole organismal level.

81 To test the validity, we will compare the ratios of whole organismal ROS production per

82 unit oxygen consumption between two hypothetical animals. This comparison can be applied to

83 individuals of the same species with different body sizes due to individual variation, or

84 individuals of the different species within a taxon, such as different mammalian species. In both

85 cases, animals' mass-specific oxygen consumption (mass-specific metabolic rate) generally

86 decreases with body size [21,22]. The conventional rate of living theory suggests, and data agree,

87 that within a taxon, the mass-specific lifetime energy expenditure of organisms is independent on

88 body mass [20,23-25]. Thus, with a few exceptions, larger animals have lower mass-specific

89 metabolic rate but longer lifespan than smaller ones. According to the most widely accepted

90 modern theory of aging, the free radical theory, the free radicals, such as ROS, are the major

91 driving force of aging. Many researchers in the field (e.g., Barja and co-workers) have shown

92 that the rate of mitochondrial ROS production rate (mtROS) is the "critical factor" for aging [1],

93 and "long-lived animals would not need to maintain high antioxidant enzyme levels, .....,

94 because they would produce mtROS at a low pace." Meanwhile, empirical data have shown that

95 the whole animal ROS production also has strong negative correlation with body size (e.g.,[1]).

96 Based on these theories and observations, it is proposed that at the whole organismal level, the

97 ROS production is proportional to the oxygen consumption. However, the challengers of the rate

98 of living theory have suggested that they are not correlated with each other, because in the 
resting state the mitochondrial ROS production rate is as large as 10 time lower than that in the 100 active state.

What the challengers of the theory focused on is the comparison between the active and

102

103

104 105

106 107 108 109 resting states. Across the respiration states, the ROS production, indeed, has weak or even no correlation with oxygen consumption rate. But the rate of living theory considers the comparison between different animals. It is unclear if, and under what condition, the disproportionality between the respiration states affects the relationship between ROS production and oxygen consumption at the whole organismal level. We will employ a simple theoretical model to investigate this question.

It is important to note that this is a conceptual model. Our purpose is to investigate whether the great difference in ROS/Oxy between the active and the resting states would break the proportionality between the ROS production and oxygen consumption at the whole organismal level. The model does not aim to simulate experiments, or fit empirical data to obtain values of certain parameters, but makes important conceptual predictions. Thus, the model does not include detailed physiological and biochemical mechanisms of mitochondrial respiration. Although simple, it offers a departure point for future theoretical models that include complex and physiologically realistic mechanisms.

The rate of oxygen consumption is regulated by the ATP requirements of the cells, which depend on the activity of the animal. We presume the resting state of mitochondria in our model to be nearly in (but never equal to) respiration state 4; in the true state 4 condition ATP synthesis ceases completely, but this only occurs during assays of isolated mitochondria. The resting state in our model refers to the in vivo state, where ATP synthesis is low but not zero. The active state is similar to respiration state 3, in which ATP synthesis rate is high and ROS/Oxy is low, 
122 compared to state 4 . In reality, mitochondria are somewhere along a continuous function

123 between two states within an organism. In this simple conceptual model, we only chose two

124 extreme states, because the difference in ROS/Oxy between these two extremes is the largest, and

125 the variation of the proportionality between the whole organismal ROS production and oxygen

126 consumption in the medium states will be bracketed by the two extreme states.

127

128

129 130 variables.

131

132

133

134

135

136

137

\section{Modeling development and Results}

We now present the key assumptions, together with definitions of the parameters and

1. Level of mitochondrial activity: One of the most important parameters in our model is the probability $k$ of a mitochondrion operating at the active state. This parameter can be interpreted in two ways. Averaging over all the mitochondria in an animal, $k$ is the proportion of time that a single mitochondrion is operating in the active state. Alternatively, $k$ can be considered as the fraction of the total mitochondria in an animal that are operating in the active state during a given period. These two interpretations are equivalent. The current general consensus is that mitochondria in vivo spend a high proportion of their time actively producing ATP [4], but the exact value of $k$ is unknown. Thus, in our model, we vary $k$ from 0 to $100 \%$.

At the whole organism level, the oxygen consumption rate at maximal rates of exercise has been found to be 3-20 times greater than that at the resting state [26]. We assume that this ratio of maximal to resting rates of oxygen consumption is of similar magnitude at the mitochondrial level, and use " $g$ " to denote it; we set $g$ to be 5.0 in our calculation.

2. Difference in ROS production per unit oxygen consumed (ROS/Oxy) between two states: ROS production is highly variable, having been found to depend on PMF, ADP 
145 availability, substrate concentrations, oxygen partial pressure, and whether the measurement is 146 conducted in isolated mitochondria or in vivo $[4,15]$. The in vivo values of $R O S$ are currently 147 little known due to technical limitations in measuring ROS production in living animals, and 148 extrapolation of absolute rates of ROS production by isolated mitochondria to the in vivo 149 situation is problematic [4]. However, our goal here is to compare ROS/Oxy between different 150 respiration states, and for this goal it is not necessary to know the absolute values of ROS 151 production. What is important is the difference (the ratio) in it between two respiration states. 152 We denote the ratio of ROS/Oxy in the active state and that in the resting state as $h$. Some studies 153 on isolated mitochondria suggested that ROS/Oxy in State 4 can be 10 -fold of that in State 3, i.e., 154 the value of $h$ is about $0.1[13,19]$. Other studies showed smaller differences between the two 155 states. In isolated mitochondria from mud clam, ROS/Oxy is twice as high as in State 4 than in 156 that in State $4(h=0.5)$ [27]. Another study on mitochondria from rat skeleton muscle showed a 157 roughly 4-fold difference $(h=0.25)$ [28]. Our interest here is to study whether the assumption of 158 "the rate of living" hypothesis - the proportionality between the whole organismal ROS 159 production and oxygen consumption [10,12]—still holds when considering the difference 160 between the resting and active states. Thus, we set $h$ to vary between 0.005 and 0.5 , so that the 161 ROS/Oxy in the resting state is $2(=1 / 0.5)$ to $200(=1 / 0.005)$ times greater than that in the active 162 state.

We now consider an animal. An average mitochondrion of the animal that operates in the 164 resting state consumes $C$ units of oxygen per unit time. The oxygen consumption rate of the 165 average mitochondrion in the active state is $g$ times higher, so the oxygen consumption in the 166 active state is $g \times C$. Recalling our first assumption, during a given period, the fractions of 167 mitochondria in this animal operating in the resting and the active states are $1-k$ and $k$, 
168 respectively, so the whole animal's total oxygen consumption $\left(O_{2, \text { whole org }}\right)$ is the weighted sum

169 of the oxygen consumptions in the two states: $O_{2, \text { whole org }}=(1-k) \times C+k \times g \times C$. In the

170 resting state, we set the value of ROS/Oxy to be $R$, and the value in the active state is therefore

$171 h \times R$. Note, $R$ and $h \times R$ are values of per unit oxygen consumed. So, for $C$ units of oxygen

172 consumed, the ROS produced in the resting state is $R \times C$, and that in the active state is $h \times R \times C$.

173 Again, the fractions of mitochondria operating in the resting and the active states are $1-k$ and $k$,

174 respectively. So the total ROS produced by all the mitochondria (the weighted sum of the resting

175 and active states) is $R O S_{\text {whole org }}=(1-k) \times R \times C+k \times g \times h \times R \times C$.

176 Thus, at the whole organismal level, the ratio of the total ROS production and the total

177 oxygen consumption, denoted as $F\left(=R O S_{\text {whole org }} / O_{2 \text {, whole org }}\right)$, can be estimated as

178

$$
\begin{aligned}
F & =\frac{(1-k) \times R \times C+k \times g \times h \times R \times C}{(1-k) \times C+k \times g \times C} \\
& =R \times \frac{(1-k)+k \times g \times h}{(1-k)+k \times g}
\end{aligned}
$$

Here, $R$ is set to be the value of ROS/Oxy in the resting state, which is a constant with an

180 arbitrary unit. As explained above, our goal is not to estimate the absolute value of $F$ and

181 compare it to empirical data. Thus, we set the constant $R$ to be 1.0 for estimating the relative

182 values. The equation above then reduces to:

183

$$
F=\frac{(1-k)+k \times g \times h}{(1-k)+k \times g} \quad \text { Eq. } 1
$$

With $g$ being a constant, the whole animal ROS production per unit oxygen consumption

$186(F)$ only depends on two parameters, $h$, the ratio of ROS per unit oxygen in the active state to 187 that in the resting state; and $k$, the fraction of the time that mitochondria operate in the active 
state. We explore the consequences of variation in these two parameters. It is straightforward to see from Eq. 1 that $F$ increases with $h$, and decreases with $k$ (Fig.1).

Showing that the whole organismal $\operatorname{ROS}_{\text {whole org }} / O_{2, \text { whole org }}$ (the value of $F$ ) decreases with activity level $(k)$ and increases with the ratio of $R O S / O x y$ between the respiration states $(h)$ is not the goal of this study, because even without a quantitative model, like ours, researchers in this field can easily reach the same but qualitative conclusion. As stated in the Introduction, our goal is to investigate if, and under what conditions, the disproportionality between the respiration states affect the relationship between the whole animal ROS production and oxygen consumption, and verify the validity of the assumption of the rate of living and oxidative stress theories.

To reach this goal, we need to compare the values of $F\left(=R O S_{\text {whole org }} / O_{2, \text { whole org }}\right.$ ) of different animals. If the animals have the same $F$, then the proportionality holds, i.e., as the whole animal oxygen consumption increases, the whole animal ROS production increases proportionally. In this case, the ratio of $F$ 's of two animals is equal to 1.0 . If this ratio is close to 1.0, then the variation in $F$ between animals is insignificant, and the whole animal ROS production is roughly proportional to the whole animal oxygen consumption. In contrast, a ratio that is far away from 1.0 indicates that for the same amount of oxygen consumption, one animal produces more ROS than the other animal, and the assumption of "rate of living" hypothesis does not hold.

To estimate the ratio of $F$ 's of animals, first, we set the $F$ of an animal with a $k$ of 0.7 as our reference value (note: $k$ varies between 0 and 1 . As a reference, it can be set at any value); And then we calculate the ratio of $F$ 's of animals with $k=0.1,0.3$, and 0.5 relative to this

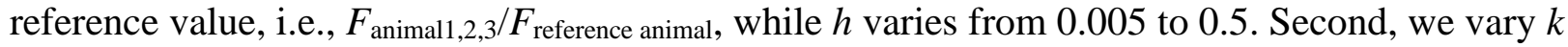


211 from 0.0 to 1.0 , and set the $F$ of an animal with a $h$ of 0.1 as the reference value; we then

212 calculate the ratio of $F$ 's of animals with $h=0.005,0.01$, and 0.5 relative to this reference value.

Figure 2A shows that if the value of ROS/Oxy in the active state is 200 times smaller than 214 that in the resting state $(h=0.005$, the left ends of the curves in Fig. 2A), then the ratio of $F$ of 215 one animal to the reference ranges from 2-fold $(k=0.5$ versus $k=0.7)$ up to 8 -fold $(k=0.1$

216 versus $k=0.7)$. For interpretation of this result, consider two animals, in both of which the

217 difference in ROS/Oxy between the resting and the active state is 200-fold (i.e. $h=0.005$ ). If the 218 mitochondria of one animal spend $10 \%$ of their time in the active state (i.e. $k=0.1$ ), and those of 219 the other animal spend $70 \%$ of their time in the active state $(k=0.7)$, then the first animal 220 produces eight times more ROS per unit oxygen consumed than does the second animal. This is 221 because the mitochondria of the first animal spend most of their time (90\%) in the resting state, 222 in which ROS/Oxy is much higher (200 times) than that in the active state. However, these are 223 extreme values for both $h$ and the difference in $k$ between the two animals. The value of 224 ROS/Oxy in the resting state is unlikely to be 200 times higher than that in the active state, and 225 the fraction of time spent in the active state of one animal is unlikely to be 7 times smaller than 226 the other animal $(k=0.1$ versus $k=0.7)$. The ratio of $F$ 's decreases both as $h$ increases and as the difference in $k$ between two 228 animals decreases. For a more realistic physiological setting, where $h=0.1$ (a value obtained 229 from empirical study of isolated mitochondria [19]), and the two animals have similar values for $230 k($ e.g. $k=0.5$ versus $k=0.7)$, the ratio of $F$ of one animal to the reference value is greatly 231 reduced from 8-fold to 1.46-fold. Moreover, the real value of $h$ can be even larger than 0.1 . 232 Studies on mitochondria isolated from mud clam [27] and rat [28] found that the ROS per unit 233 oxygen in the resting state is 2 - and 4-fold of that in the active state, respectively $(h=0.5$ and 
2340.25 ). Once $h$ is above 0.1 , the ratio is very close to 1.0 , as indicated by shallow gradients for the 235 curves in Fig. 2A. Thus, with these realistic physiological parameters, we consider that ROS236 oxygen proportionality at the whole organismal level generally holds.

237 Figure 2B shows the ratio of $F$ 's for three pairs of animals with different $h$ values $(h=$ $2380.005,0.01,0.05$ versus $h=0.1$ ), as $k$ varies. The ratio of $F^{\prime}$ s is almost independent on $h$ at low239 medium values of $k$ : for $k<0.7$, the ratio of $F$ 's only ranges from 1.0 to 1.5 -fold, even when 240 comparing two animals with very different values of $h$ (e.g. $h=0.005$ versus 0.1 ; black curve in 241 Fig. 2B). However, the effect of the difference in $h$ 's between animals on the ratio of $F$ 's 242 becomes increasingly important, as $k$ approaches 1.0.

\section{Discussion}

Four conditions for the whole organismal proportionality

Our model suggests that, different from the claims by the challengers of the "rate of

247 living" hypothesis (e.g., [1]), the difference in ROS/Oxy between the resting and the active states

$248(h \neq 1)$ does not necessarily cause the disproportionality between the whole animal ROS

249 production and oxygen consumption. It depends on the values of $k$ (the probability of a mitochondrion operating in the active state, which equivalent to the fraction of time it is in this

251 state) and $h$ (the difference in ROS/Oxy between the respiration states) with $k$ playing a more 252 important role.

The blue curve in Fig. 2A shows that if two animals have the same $h$ value that is larger 254 than 0.05 , and the $k$ values of theirs are similar $(k=0.5$ versus $k=0.7)$, the ratio of $F$ 's between 255 the two animals is smaller than 1.5, even if the ROS/Oxy in the active state is 20 times lower than 256 that in the resting state $(h=0.05)$. When $h=0.5$ (a value found in some empirical studies), the 
257 ratio of $F$ 's is insignificantly different than 1.0. So, in the case of the blue curve in Fig.1, the 258 whole organismal ROS production is virtually proportional to oxygen consumption for a wide 259 range of $h$ values. This means that ROS/Oxy can be very different between respiration states, but 260 the whole organismal proportionality still holds. Thus, the first condition for the whole organismal proportionality is that animals under 262 comparison have the same $h$ value that is larger than 0.05 and similar values of $k$. The smallest 263 value of $h$ found in the empirical studies is 0.1 . Also, empirical data suggest that the variation of $264 h$ between animals is small. For example, $h$ of mud clam is 0.5 [27] and $h$ of rat muscle is 0.25 265 [28], i.e., 2-fold difference between two species from very different taxon groups. So, we assume 266 that the difference in $h$ of the animals from the same taxon is insignificant. Thus, regarding the 267 first condition, the values of $k$ are the dominating factor. Excluding the extreme comparisons, 268 such as extreme active versus sedentary individual animals or animal in torpor versus pregnant 269 animals, the condition of "similar $k$ " is physiologically realistic, especially for the animals of the 270 same species, which live in the same niche, and have similar level of energy demand. Moreover, 271 it has been found that animals within a taxon, such as mammals or birds, generally have similar 272 field active scope (the ratio of field and resting metabolic rate) [21], indicating that they have 273 similar relative activity level. Thus, it is reasonable to assume that they have similar $k$ values too. 274 The first condition is sufficient but necessary, as the red curve in Fig.2A shows the 275 second condition for the proportionality. If the $k$ values of animals are not similar (e.g., $k=0.3$ 276 v.s. 0.7$)$, as long as the $h$ value is large enough $(>0.5)$, the ratio of $F$ 's is still insignificantly close 277 to 1.0. However, this condition may not be realistic, because, as far as we are concerned, the 278 largest empirical value of $h$ was found to be 0.5 [27]. 
The blue curve in Figure 2B suggests the third condition for the proportionality, which is

280 similar to the first condition. If two animals have the same $k$ values, and similar $h$ values (e.g., $h$

$281=0.05$ v.s. 0.1 in the blue curve), the ratio of $F$ 's is close to 1.0. As we discussed above, this

282 condition is physiologically possible, especially for animals of the same species.

The third condition is also sufficient but necessary, as the red and black curves in Fig $2 \mathrm{~B}$

284 suggest the fourth condition. The curves show that even if the $h$ values of animals under

285 comparison is very different (such as 20 -fold, $h=0.005$ v.s. 0.1 , the black curve), for a large

286 range of $k$, from 0 to $\sim 0.6$, the ratio of $F$ 's between two animals is still close to $1.0(<1.5)$. Thus,

287 the fourth condition is that the animals have the same value of $k$ that is smaller than 0.6. This is

288 also a sufficient but not necessary condition. Very few, if any, empirical studies have

289 investigated how mitochondria allocate their time between operating in the resting versus the

290 active state (the $k$ value), and how this varies with physiological demands or environmental

291 conditions. Recalling that $k$ is the proportion of time that a single mitochondrion operates in the

292 active state, averaging over all the mitochondria in an animal during a given period, or

293 equivalently the fraction of the total mitochondria in an animal that operates in the active state

294 during a given period. Although no empirical data is available for verification, it is possible that

295 for animals that are not under continuous high energy demands, such as lactating, during any

296 given period an average mitochondrion does not allocate more than $60 \%$ of its time in the active

297 state, or no more than $60 \%$ of the total mitochondria operating in the active state $(k<0.6)$. We

298 call for future research to investigate this question.

299 Together, these four conditions highlight the importance of the parameter $k$ : As long as

300 the animals under comparison have similar $k$ values, but do not have to be the same, which are

301 lower than a certain value (our model suggests the value to be 0.6), then no matter how different 
302 the ROS/Oxy between the active and resting states is (how large the $h$ value is), even if it is as

303 large as 200 -fold $(h=0.005)$, the whole organismal proportionality virtually holds, opposite of

304 the suggestion from the challengers of the rate of living theory. Again, it is worth to note that

305 very small value of $h$, such as 0.005 is physiologically unrealistic, because the smallest $h$ value

306 found in empirical study is $h=0.1$ (10-fold difference between the states) $[13,19]$. Thus, the $k$

307 values of animals under comparison and how close they are the critical factors of the whole

308 organismal proportionality.

309 It is possible that for a given energy demand, the time budget of mitochondria deviates

310 from that which would minimize ROS production due to other constraints or tradeoffs. For

311 instance, minimizing ROS is unlikely to be of prime importance in semelparous species during

312 their single breeding season, since their fitness is unaffected by any oxidative damage that would

313 only have effects over the long term. It is also worth mentioning that high levels of exercise

314 would shift mitochondria towards the active state, but also increase ROS production defenses at

315 the same time. Quantitative studies on the arms race between the positive and negative effects of

316 exercise, however, remain to be performed.

The effects of mitochondrial uncoupling on the proportionality

It has been shown across a diversity of organisms (including snail, lizard, rat and horse

319 [18]) that the degree of uncoupling, the fraction of oxygen consumption spent on offsetting the

320 proton leak, ranges from $15-25 \%$ (in the mitochondria of cells from snail hepatopancreas) to

$32135 \sim 50 \%$ (in the mitochondria of rat muscle) with an average of $20 \%$. These values are the

322 averages over the mitochondria operating at different states; the level of uncoupling is usually

323 lower in the active state than in the resting state [18]. 
Uncoupling may affect the proportionality of the whole animal ROS production and oxygen consumption through two different mechanisms. First, as explained in the Introduction section, uncoupling reduces ROS production by reducing membrane potential. However, the uncoupling-induced reductions in ROS production are different in the resting and the active states. ROS production is more sensitive to membrane potential in the resting state than it is in the active state. So, the same degree of uncoupling in the active state causes relatively less reduction in ROS production, compared to that in the resting state [19]. ROS/Oxy in the active state is lower than that in the resting state [13,19,27,28], and uncoupling reduces the ROS/Oxy difference between the two states. Recalling that in our model a declination of the ROS/Oxy difference between two respiration states is indicated by an increasing $h$, thus uncoupling makes the value of $h$ larger. Fig. 2A shows that the variation in $F$ across animals (the ratio of $F$ ) decreases as $h$ increases. Thus, our theoretical model suggests that, if everything else kept the same, the mitochondrial uncoupling reduces the variation in $F$ across animals, and therefore strengthens the proportionality between the whole organismal ROS/Oxy.

Second, since uncoupling reduces the ATP synthesis rate, it is possible, although we are not aware of empirical evidence, that to meet the ATP demand of animals, mitochondria may spend more time in the active state, where the ATP synthesis rate is high. This means that uncoupling may increase the value of $k$. However, increasing $k$ may not necessarily affect the whole organismal proportionality. Fig. 2A shows that if two animals have the same value of $h$, the ratio of $F^{\prime} s$ of them increases as the difference in their $k$ 's increases (instead of $k$ itself). It is possible that the same degree of uncoupling in two animals, especially animals of the same species, increases their $k$ 's to the same degree, so that the difference in their $k$ 's keeps unchanged. In this case, the ratio of $F$ 's is not affected by uncoupling. 
Nonetheless, if two animals have different $h$ values, Fig. 2B shows that an increase in $k$ does cause an increase in the ratio of $F$ 's. It is important to note, however, the curves shown in Fig. 2B include the cases, where the differences in $h$ 's between two animals are very large, $h=$ 0.005 v.s. 0.1 ( 20 -fold), and $h=0.01$ v.s. 0.1 (10-fold), which are unrealistic, especially for the animals of the same species. More likely, the difference in $h$ 's is much smaller than those values, and uncoupling may not enlarge the difference greatly. So, a more physiologically realistic curve with a smaller difference in $h$ will be around or even below the blue curve in Fig. 2B ( $h=0.05$ v.s. 0.1, 2-fold difference). In such a curve, even a large $k(>0.7)$ does not offset the proportionality too much.

\section{Conclusion}

The assumption of proportionality between ROS production and oxygen consumption at the whole organismal level is one of the fundamental pillars of the rate of living theory and the oxidative stress theory, and plays important roles in the study of aging, such as developing theoretical models $[5,29,30]$, and interpreting the results of experiments [7,31]. Thus, the utility of this model lies in its contribution to conceptually clarifying this controversial issue in the field. Our model considers only two extreme mitochondrial respiration states. A quantitative model that aims to mimic the real mitochondrial respiration, and simulate experiments would consider continuous states of mitochondrial respiration between the two extremes. Moreover, our model assumes static states. For example, the two key parameters in the model are fixed constants during a given period. In reality, they vary with animal's ATP demand, activity level, and other factors, such as aging. So, a more realistic model would consider the dynamic state functions of time, which will lead to first or second order differentials. 
371 oxygen consumption across individual animals depends on two parameters, the fraction of the

372 time that mitochondria operate in the active state (the $k$ value) and the difference in ROS

373 production per unit oxygen consumed between the active and that in the resting state (the $h$

374 value), with the former affecting the variation more heavily than the latter. The model suggests

375 that under four conditions, three of which are physiologically possible, the difference between

376 the respiration states (the $h$ values) does not upset the proportionality between whole animal

377 ROS production and oxygen consumption. Finally. the model suggests that in general the

378 mitochondrial uncoupling makes the correlation between ROS production and oxygen

379 consumption more proportional.

381 The authors declare no conflict of interest.

References:

1 Barja G. 2013. Updating the Mitochondrial Free Radical Theory of Aging: An Integrated View, Key Aspects, and Confounding Concepts. Antioxidants \& redox signaling 19: 1420-45.

2 Sohal RS, Orr WC. 2012. The redox stress hypothesis of aging. Free Radical Biology and Medicine 52: 539-55.

3 Metcalfe NB, Alonso-Alvarez C. 2010. Oxidative stress as a life-history constraint: the role of reactive oxygen species in shaping phenotypes from conception to death. Functional Ecology 24: 984-96.

$4 \quad$ Murphy M. 2009. How mitochondria produce reactive oxygen species. Biochem J 417: 1-13.

$5 \quad$ Hou C, Amunugama K. 2015. On the complex relationship between energy expenditure and longevity: Reconciling the contradictory empirical results with a simple theoretical model. Mechanisms of Ageing and Development 149: 50-64.

6 Ristow M, Zarse K. 2010. How increased oxidative stress promotes longevity and metabolic health: The concept of mitochondrial hormesis (mitohormesis). Experimental Gerontology 45: 410-8.

7 Salin K, Auer SK, Rey B, Selman C, et al. 2015. Variation in the link between oxygen consumption and ATP production, and its relevance for animal performance.

8 Munro D, Treberg JR. 2017. A radical shift in perspective: mitochondria as regulators of reactive oxygen species. Journal of Experimental Biology 220: 1170-80.

9 Salin K, Villasevil EM, Anderson GJ, Auer SK, et al. 2018. Decreased mitochondrial metabolic requirements in fasting animals carry an oxidative cost. Functional ecology 32: 2149-57. 
Sohal RS, Mockett RJ, Orr WC. 2002. Mechanisms of aging: an appraisal of the oxidative stress hypothesis. Free Radical Biology and Medicine 33: 575-86.

11 Speakman JR, Talbot DA, Selman C, Snart S, et al. 2004. Uncoupled and surviving: individual mice with high metabolism have greater mitochondrial uncoupling and live longer. Aging Cell 3: 87-95.

12 Hulbert AJ, Pamplona R, Buffenstein R, Buttemer WA. 2007. Life and Death: Metabolic Rate, Membrane Composition, and Life Span of Animals. Physiological Reviews 87: 1175-213.

13 Nicholls DG. 2004. Mitochondrial membrane potential and aging. Aging Cell 3: 35-40.

14 Speakman JR, Garratt M. 2014. Oxidative stress as a cost of reproduction: beyond the simplistic trade-off model. Bioessays 36: 93-106.

15 Kadenbach B. 2003. Intrinsic and extrinsic uncoupling of oxidative phosphorylation. Biochimica et Biophysica Acta (BBA)-Bioenergetics 1604: 77-94.

16 Dzbek J, Korzeniewski B. 2008. Control over the contribution of the mitochondrial membrane potential (DeltaPsi) and proton gradient (DeltapH) to the protonmotive force (Deltap). In silico studies. J Biol Chem 283: 33232-9.

17 Barja G. 2007. Mitochondrial Oxygen Consumption and Reactive Oxygen Species Production are Independently Modulated: Implications for Aging Studies. Rejuvenation Research 10: 215-24.

18 Brand MD. 2000. Uncoupling to survive? The role of mitochondrial inefficiency in ageing. Experimental Gerontology 35: 811-20.

19 Korshunov SS, Skulachev VP, Starkov AA. 1997. High protonic potential actuates a mechanism of production of reactive oxygen species in mitochondria. FEBS Letters 416: 15-8.

20 Pearl R. 1928. The rate of living: Alfred A. Knopf, New York.

21 Nagy KA, Girard IA, Brown TK. 1999. Energetics of free-ranging mammals reptiles and birds. Annual Review of Nutrition 19: 247-77.

22 Savage VM, Gillooly JF, Woodruff WH, West GB, et al. 2004. The predominance of quarterpower scaling in biology. Functional Ecology 18: 257-82.

23 Rubner M. 1908. Das Problem der Lebensdauer und seine Beziehungen sum Wachstum und Ernahrung Munich: Oldenbourg.

24 McCoy MW, Gillooly JF. 2008. Predicting natural mortality rates of plants and animals. Ecology Letters 11: 710-6.

25 Speakman JR. 2005. Body size, energy metabolism and lifespan. Journal of Experimental Biology 208: 1717-30.

26 Weibel ER, Hoppeler H. 2005. Exercise-induced maximal metabolic rate scales with muscle aerobic capacity. Journal of Experimental Biology 208: 1635-44.

27 Abele D, Heise K, Pörtner H-O, Puntarulo S. 2002. Temperature-dependence of mitochondrial function and production of reactive oxygen species in the intertidal mud clam Mya arenaria. Journal of Experimental Biology 205: 1831-41.

28 Venditti P, Bari A, Di Stefano L, Di Meo S. 2009. Effect of T3 on metabolic response and oxidative stress in skeletal muscle from sedentary and trained rats. Free Radical Biology and Medicine 46: 360-6.

29 Hou C. 2013. The energy trade-off between growth and longevity. Mechanisms of Ageing and Development 134: 373-80.

30 Drenos F, Kirkwood TBL. 2005. Modelling the disposable soma theory of ageing. Mechanisms of Ageing and Development 126: 99-103.

31 Metcalfe NB, Monaghan P. 2013. Does reproduction cause oxidative stress? An open question. Trends in Ecology \& Evolution 28: 347-50. 

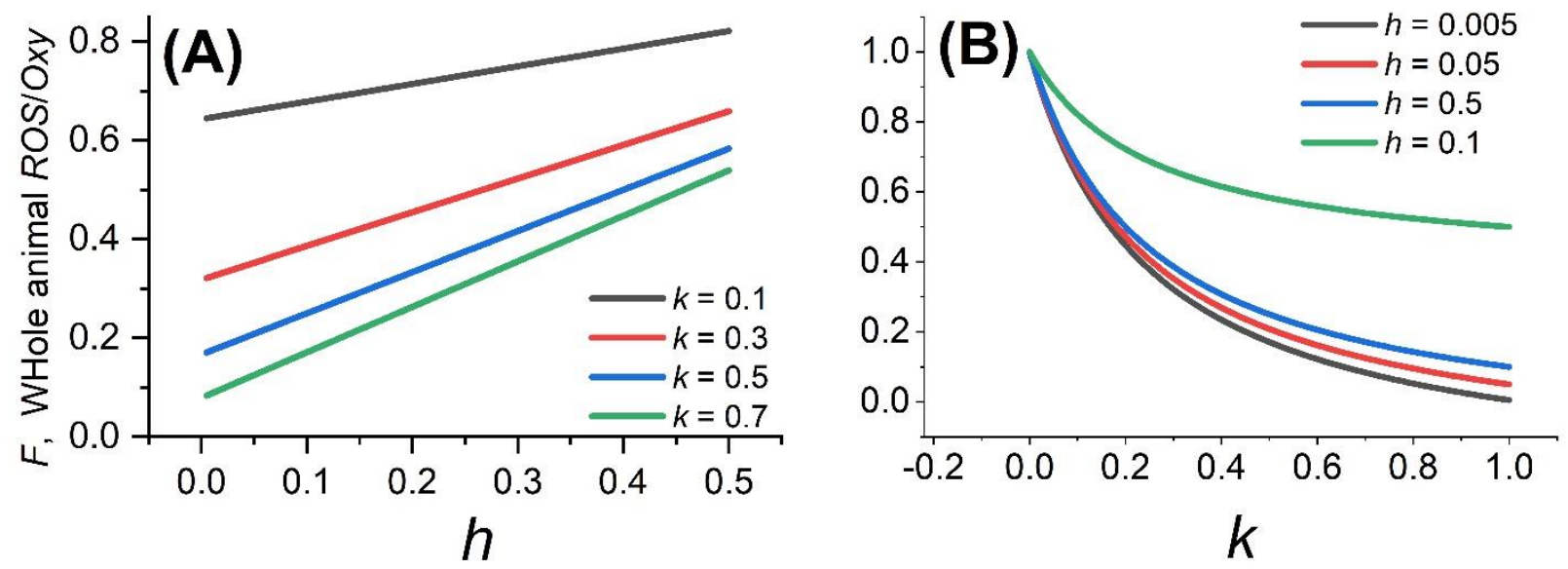

450

451

452

453

454

455

456

457

458

459

460

461

Figure 1. The whole organismal ROS production per unit oxygen consumed, $F$, as a function of $h$ (Fig.1A), and $k$ (Fig.1B).
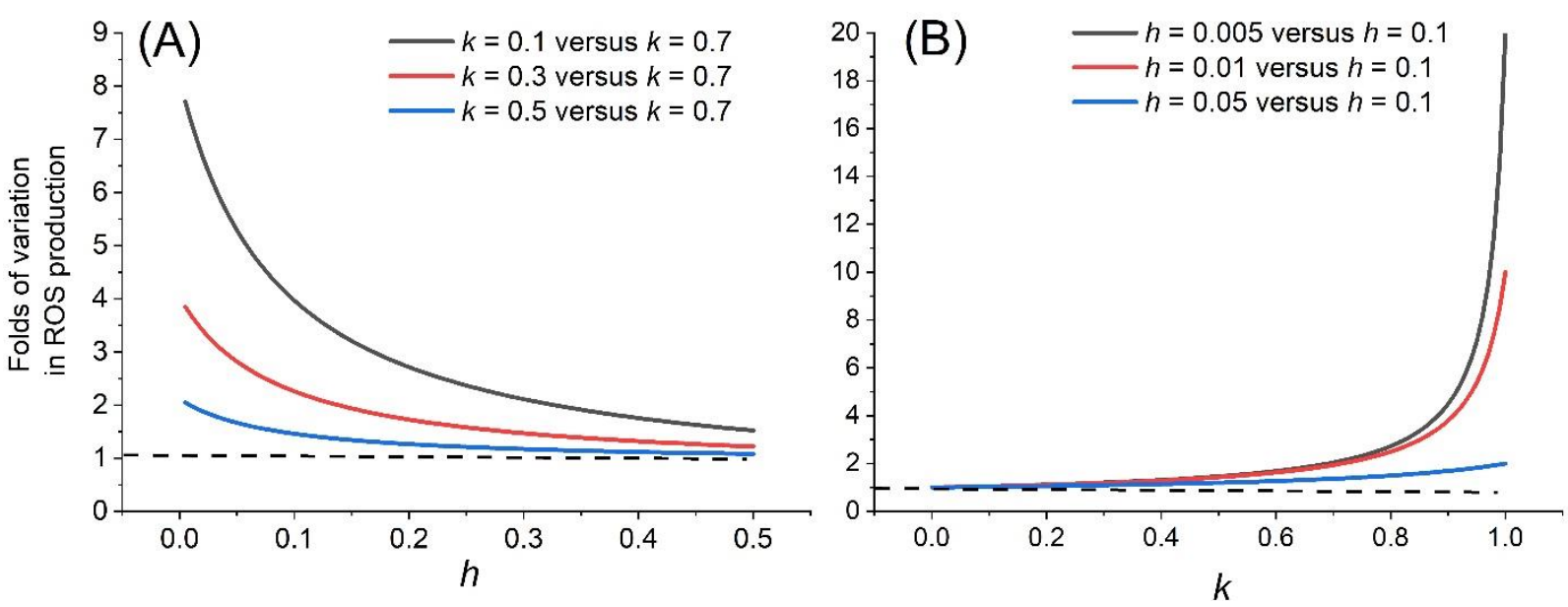

Figure 2. The ratio of $F$ 's (the whole organismal ROS production per unit of oxygen

consumed) between two animals. (A) The ratio in three pairs of animals as a function of $h$. In

each pair, two animals have different values of $k$; (B) The ratio in three pairs of animals as a

function of $k$. In each pair, two animals have different values of $h$. Curves illustrate the ratios of

$F$ 's of two animals with different parameter values, e.g., the black line in panel (A) expresses the

$F$ ratio of an animal with $k=0.1$ relative to the one with $k=0.7$. The dashed horizontal lines

indicate $F=1.0$ (perfect proportionality). See text for further explanation. 